\title{
UMA REVISÁO SISTEMÁTICA DA LITERATURA SOBRE M-LEARNING, ABP E MODELAGEM MATEMÁTICA NA APRENDIZAGEM DE CDI EM ENGENHARIAS
}

\author{
Fulvio Bianco Prevot ${ }^{1}$ \\ Juliano Schimiguel ${ }^{2}$
}

\begin{abstract}
Resumo: Pesquisas indicam que boa parte dos alunos dos cursos de Engenharia, no Brasil, não apresentam bom desempenho nos cursos de Cálculo Diferencial e Integral (CDI), devido a alguns fatores, dentre eles a precária base matemática, trazida dos ensinos fundamental e médio, bem como os conteúdos apresentarem relativo grau de abstração. Nas Instituiçôes de Ensino Superior, mesmo com a disponibilização de estratégias de acompanhamento e auxílio aos alunos (plantóes de dúvida, monitorias), discentes com maiores problemas de aprendizagem ainda parecem apresentar muitas dificuldades. Este artigo apresenta uma revisão sistemática da literatura (RSL), no que tange à aplicação da Aprendizagem Baseada em Problemas (ABP, ou, em inglês, PBL), da Modelagem Matemática (MM), da m-learning (fundamentada na Teoria da Atividade - TA) e ao uso de smartphones e aplicativos matemáticos, no contexto do ensino e da aprendizagem de Cálculo Diferencial e Integral (CDI) em cursos de Engenharia, no período de 2005 a 2019. A metodologia para a RSL baseia-se nos trabalhos de Kitchenham (2004) e no modelo proposto por Almeida (2016). Descrevem-se algumas contribuiçóes que o emprego destas metodologias e tecnologias têm trazido ao ensino de CDI nos cursos mencionados. Percebe-se, no resultado da pesquisa, um pequeno, porém crescente, número de pesquisas envolvendo estes temas, o que leva a inferir que ainda há espaço para novas pesquisas neste campo, no sentido aperfeiçoar os processos de ensino e de aprendizagem relacionados.
\end{abstract}

Palavras-chave: Educação em Engenharia, ABP (PBL), Modelagem Matemática, Cálculo, M-Learning, Apps Matemáticos.

1 Doutor em Ensino de Ciências e Matemática, Mestre em Engenharia Espacial e Controle, Engenheiro Eletricista, Licenciado em Ciências-Matemática, Licenciado em Pedagogia Professor EBTT do Instituto Federal de Educação, Ciência e Tecnologia de São Paulo - Campus Sáo Paulo.

2 Doutor e Mestre em Ciência da Computação, Bacharelado em Informática - Professor Titular III do Programa de Doutorado / Mestrado em Ensino de Ciências e Matemática - Universidade Cruzeiro do Sul. 


\title{
A SYSTEMATIC REVIEW OF LITERATURE ON M-LEARNING, PBL AND MATHEMATICAL MODELING IN CDI LEARNING IN ENGINEERING
}

\begin{abstract}
Many Engineering courses' students do not reach approval on disciplines that teach Calculus contents, due to some factors, such as precarious mathematical basis, brought from previous steps of formal education, even when traditional aiding strategies are offered (doubt-resolving shifts, monitoring) by Higher Education Institutions to students presenting greater learning problems in the mentioned contents. This paper proposes a systematic review (SR) of literature, regarding the application of Problem-Based Learning (PBL), Mathematical Modeling (MM), M-Learning (grounded on Activity Theory - AT) and the use of smartphones and mathematical apps, in the context of teaching and learning of Differential and Integral Calculus in Engineering courses in the elapsed period between 2005 and 2019. The research methodology, involved on SR, stands on the works of Kitchenham (2004) and the model proposed by Almeida (2016). This paper describes some contributions to related teaching and learning processes, brought by application of these methodologies and technologies. The results show a small but growing number of works incorporating these themes, which leads to infer that there is still room for further research in this field, in order to improve the mentioned processes.
\end{abstract}

Keywords: Engineering Education, PBL, Mathematical Modeling, Calculus, M-Learning, Mathematical Apps.

\section{INTRODUÇÃO}

De acordo com Kidron (2014), o Cálculo Diferencial e Integral (CDI) é considerado como uma das grandes criaçôes da Matemática. Em muitos países, é um conteúdo ensinado desde o Ensino Médio até a Universidade. A aprendizagem de CDI inclui a análise de problemas, nos quais ocorrem variaçóes, movimentos ou oscilaçóes, o que requer conhecimento prévio de conceitos fundamentais, como, por exemplo, variáveis e funçóes, a fim de que se possa compreender os conceitos que embasam o CDI, tal como o conceito fundamental de limite e processos limitantes, que implicitamente contêm variaçôes de quantidades. Para compreender o conceito matemático de limite, é necessária muita abstração, um recurso mental tipicamente exigido em matemática mais avançada (PREVOT, 2019).

Os conteúdos e as aplicaçóes de CDI são de extrema importância para a formação do Engenheiro, em qualquer uma das especialidades de Engenharia existentes no mercado de trabalho. Em conjunto com a Física, a Geometria Analítica e outros componentes do ensino básico, CDI propóe prover o aluno de ferramental matemático e analítico, para analisar problemas, estabelecer alternativas de solução, criar modelos que ajudam a entender o problema e deliberar a solução mais viável para a situação estabelecida (PREVOT, 2019). Além disso, os conteúdos de CDI são básicos para outros componentes curriculares dos núcleos de conteúdos profissionalizantes e específicos dos currículos dos cursos de engenharia.

Jaworski (2013) destaca as seguintes competências matemáticas: 
a) A habilidade de fazer e a responder questóes com e na matemática: pensar matematicamente, raciocinar matematicamente, propor e solucionar problemas matematicamente e modelar matematicamente;

b) A habilidade de lidar com ferramentas e linguagem matemáticas: representar entidades matemáticas, manipular formalismo e símbolos matemáticos, comunicar-se matematicamente e fazendo uso de ajudas e ferramentas.

Segundo Niss (2003, apud JAWORSKI, 2013), possuir competência matemática, significa ter o conhecimento, compreender, fazer e usar matemática e ter uma opiniáo bem fundamentada a respeito, em uma variedade de situaçóes e contextos, nos quais a matemática tenha ou possa um papel essencial.

Desta forma, os estudantes de Engenharia precisam não apenas serem aprovados em CDI, mas principalmente, desenvolverem estudos neste componente curricular que os conduzam à formação de uma base conceitual sólida e um conjunto de competências (incluindo as competências matemáticas), tendo em vista o prosseguimento de estudos que levam à formação do Engenheiro.

Alguns fatores podem contribuir para um indesejável resultado (reprovação, por exemplo), por parte dos alunos dos cursos de Engenharia no componente Cálculo Diferencial e Integral (CDI), dentre eles a precária base matemática, trazida dos ensinos fundamental e médio. Segundo Ferruzzi e Almeida (2013), a falta de vivência em aplicaçóes de CDI, na área de Engenharia, "[...] tem mostrado, ao longo do tempo, a dificuldade dos alunos na compreensão e aplicação dos conteúdos teóricos matemáticos em seu campo profissional e na sua vida diária." Nestas condiçôes, parece que muito raramente um estudante consegue acompanhar o curso desta disciplina, mesmo quando recorre a ajuda externa (aulas particulares, monitoria, plantôes de dúvidas ou outros). Nas Instituiçóes de Ensino Superior, mesmo com a disponibilização de estratégias de acompanhamento e auxílio aos alunos (plantôes de dúvida, monitorias), discentes com maiores problemas de aprendizagem ainda parecem apresentar muitas dificuldades (PREVOT, 2019).

Conforme abordado mais adiante, seja pela resistência de muitos docentes em tentar incorporar em seu trabalho novos métodos e / ou tecnologias, talvez por receio de não concluir o planejamento de ensino dentro do período letivo oficial, ou por falta de tempo, para se preparar ou por problemas de acesso aos meios, seja por dificuldades ou entraves institucionais, ou ainda por estarem muito presos a metodologias tradicionais, perde-se a oportunidade de se utilizar recursos tecnológicos de ponta e / ou metodologias que possam despertar o interesse e melhorar a motivaçáo dos alunos em querer assimilar conteúdos que requeiram maior grau de abstração e concentração nos estudos.

Há professores de componentes curriculares presenciais que tomam iniciativas de colocar à disposiçáo dos alunos, materiais suplementares em ambientes virtuais de aprendizagem (AVA) ou mesmo em "sites" pessoais, tais como resumos e listas adicionais de exercícios, que os estudantes têm a opção de consultar e utilizar, porém 
estes últimos ainda carecem de uma orientação e atendimento mais efetivos, a fim de sanar suas dúvidas.

Desta forma, percebe-se que os alunos, especialmente os que têm problemas de desempenho em CDI, poderiam dispor de metodologia, associada ao poder dos meios eletrônicos disponíveis, que complementem a metodologia e trabalho desenvolvido pelo docente nas aulas regulares. Por exemplo, o uso de metodologias ativas, tais como a Aprendizagem Baseada em Problemas, a Teoria da Atividade e a Modelagem Matemática, da m-learning (Mobile Learning - Aprendizagem Móvel) e de softwares matemáticos instalados em dispositivos móveis (tablets, smartphones) poderiam tornar-se fatores de aumento da motivaçáo e interesse dos alunos, para aprender os conteúdos de CDI, bem como tornar esta aprendizagem mais atraente, mais interessante e mais dinâmica, uma vez que, através do m-learning, os estudantes poderiam ter acesso a recursos e conteúdos em qualquer hora e lugar, desde que disponíveis na rede mundial e desde que haja uma razoável conexáo wiff.

Conforme abordado mais adiante, a literatura mostra ainda poucas pesquisas com resultados de aplicaçáo desses elementos, seja isoladamente e / ou combinados entre si, no ensino de CDI em cursos de Engenharia.

Este artigo apresenta uma revisão sistemática da literatura (RSL), no que tange à aplicação da Aprendizagem Baseada em Problemas (ABP, ou, em inglês, $\mathrm{PBL}$ ), da Modelagem Matemática (MM), da m-learning (fundamentada na Teoria da Atividade - TA) e ao uso de smartphones e aplicativos matemáticos, no contexto do ensino e da aprendizagem de Cálculo Diferencial e Integral (CDI) em cursos de Engenharia, no período de 2005 a 2019.

\section{MOTIVAÇÁO E JUSTIFICATIVA}

A priori, o que motiva e justifica o desenvolvimento deste trabalho de pesquisa é a grande importância que uma aprendizagem profícua dos conteúdos de CDI possui na formação das competências relacionadas ao pensamento matemático e à capacidade de analisar e solucionar problemas, que um engenheiro deve construir ao longo do curso de Engenharia.

Porém, especificamente nesse componente curricular, historicamente existem altos índices de evasão e retenção (SILVA, JARDIM e CARIUS, 2016). Alguns pesquisadores atribuem este resultado indesejável ao pouco ou nenhum domínio dos fundamentos matemáticos necessários (por exemplo, funçôes e destreza algébrica) que os alunos dos períodos iniciais dos cursos de Engenharia trazem das etapas anteriores do ensino fundamental e médio. Outros apontam para as práticas tradicionais de ensino, nas quais o aluno é um elemento passivo, centradas no professor e calcadas normalmente na exposição e em exercícios de repetição e fixação de técnicas algébricas de desenvolvimento e soluçáo de expressôes, equaçôes, inequaçóes ou similares, com pouco enfoque nos conceitos envolvidos, em seu significado e em sua conexão com situaçôes reais. Isto pode ser um fator que desmotiva o aluno, principalmente quando a compreensão e assimilação desses 
conteúdos requerem um grau um pouco mais elevado de concentração e abstração (PREVOT, 2019).

Assim, trabalhos de pesquisa levantados nesta revisão bibliográfica, pelo menos nos últimos quinze anos, preocupam-se em propor metodologias ativas (centradas nos alunos), visando o ensino e aprendizagem de matemática, e, neste grupo, o ensino e aprendizagem de CDI, incorporando ou náo de recursos das TIC nesses processos.

Nesta mesma linha, existe o m-learning (Mobile Learning - aprendizado por dispositivos móvel), que é uma modalidade de e-learning, no qual são usados dispositivos móveis, tais como tablets e smartphones. Uma das grandes vantagens desta modalidade de ensino é a mobilidade, ou seja, o aluno não precisa de uma instalação fixa com um microcomputador; o estudante pode estar em qualquer lugar que tenha Internet disponível, tendo em vista que smartphones são dispositivos muito comuns e familiares, em especial, para as novas gerações de estudantes e que podem ser de grande valia para servir de elemento de apoio aos processos de aprendizagem dos estudantes.

Além disso, muitas empresas e entidades ao redor do mundo têm se empenhado a criar aplicativos (apps) para atender necessidades em diversas áreas do conhecimento, como, por exemplo, Física, Química, Biologia, Linguagens e Matemática. No caso desta última, uma rápida pesquisa na Internet ou em sites de vendas de apps (por exemplo, a "Play Store") mostrará dezenas ou até centenas de aplicaçóes voltadas para o ensino de Matemática, em diferentes níveis de ensino, desde o básico (operaçóes fundamentais, expressóes aritméticas, equaçóes e inequaçóes, geometria analítica, entre outros) até o superior (limites, derivadas, integrais), muitos deles gratuitos e que facilmente podem ser baixados e instalados em dispositivos móveis.

Diante de todas as ofertas proporcionadas pelas Tecnologias da Comunicação e Informação (TIC), em especial o m-learning e os apps matemáticos, uma combinação destes elementos, com abordagens metodológicas baseadas em teorias robustas, como por exemplo, a Aprendizagem Baseada em Problemas (ABP), Teoria da Atividade (TA), Modelagem Matemática (MM), pode levar o aluno a complementar os ensinamentos obtidos na aula presencial tradicional, no ensino híbrido, ou mesmo no $\mathrm{EaD}$, de maneira que entenda melhor os propósitos do que ele está a aprender em CDI, bem como porque deve adquirir tais conhecimentos, dentro de um contexto de aplicaçáo em problemas reais ou muito próximos da realidade (PREVOT, 2019).

A Aprendizagem Baseada em Problemas (ABP) ou, em inglês, Problem-Based Learning (PBL), é uma metodologia de aprendizagem relativamente recente, criada e sistematizada em meados da década de 1960 na Escola de Medicina da Universidade McMaster, na cidade de Hamilton, província de Ontário, no Canadá. Pouco depois, instituiçóes de ensino de outros países, tais como Holanda, Dinamarca, Austrália e Estados Unidos, passaram a adotar, mesmo que parcialmente, a ABP. Devido a trabalhos bem sucedidos nos cursos de Medicina, o método foi adotado por escolas 
de Engenharia, Arquitetura, Enfermagem, Direito, Pedagogia e Administração (PREVOT, 2019).

Para Souza e Schimiguel (2014), a ABP, que possui suas raízes na teoria do conhecimento do filósofo americano John Dewey (precursor de movimentos como a Escola Nova e o movimento ativista), é uma metodologia ativa de construção da aprendizagem, baseada no estudo de casos e problemas, que são utilizados como recurso que estimula à aquisição de conhecimento e compreensão de conceitos.

"Os professores expóem uma situaçáo problema para estudo aos alunos. Em
seguida, reunidos em grupos de trabalho, identificam o problema investigam,
debatem, interpretam e produzem possíveis soluçóes e demonstraçóes,
sugerindo novos caminhos para resoluçáo (SOUZA e SCHIMIGUEL,
2014)."

Já a Modelagem Matemática (MM), de acordo com Biembengut (2009), pode ser definida "[...] como processo para descrever, formular, modelar e resolver uma situação problema de alguma área do conhecimento", que aparece na literatura, por volta do início do século XX, nas áreas de Engenharia e Economia. Segundo Bassanezi (2011) a MM é um processo dinâmico, utilizado para a obtenção e validação de modelos matemáticos. É uma forma de abstração e generalização, com a finalidade de fazer previsóes e de determinar e estudar tendências. Portanto, a modelagem matemática transforma situações reais em problemas matemáticos. "A modelagem é eficiente, a partir do momento que nos conscientizamos que estamos sempre trabalhando com aproximaçóes da realidade, ou seja, que estamos elaborando sobre representaçóes de um sistema ou parte dele (BASSANEZI, 2011)."

Enfim, a Teoria da Atividade (TA), baseada nos trabalhos de Marx e Engels, desenvolvida por Leontiev, Luria e Engeström, com raiz históricocultural na psicologia soviética e nas ideias desenvolvidas por Vygotsky (mediação, internalização e desenvolvimento das funçôes mentais superiores), defende que o desenvolvimento do homem se dá pela necessidade de uma relaçáo com o meio em que está inserido, com a satisfação de alguma necessidade pessoal. Estes autores afirmam que o desenvolvimento das funçôes psíquicas decorre de um processo de apropriação de algum saber, transformando a atividade externa em atividade interna e que a mediação entre o sujeito e o ambiente ocorre por meio da linguagem (LEONTIEV, 1978; VIGOTSKII, LURIA e LEONTIEV, 2010). Leontiev (2004) estabeleceu que "[...] Por atividade, designamos os processos psicologicamente caracterizados por aquilo a que o processo (seu objeto), como um todo, se dirige, coincidindo sempre com o objetivo que estimula o sujeito a executar esta atividade, isto é, o motivo". Desta forma, pode-se entender que a aprendizagem é uma atividade humana, movida por um objetivo e por um motivo (no campo escolar, o motivo para aprender). Nesta linha de pensamento, a aprendizagem é uma atividade humana movida por um objetivo, a qual concebe três pontos de relevância: acontece em um meio social, através de uma atividade mediada nas relaçóes entre os sujeitos e é uma atividade entre o sujeito e o objeto de aprendizagem (GRYMUZA e REGO, 2014). O que diferencia uma atividade de outra é o motivo, o qual impulsiona uma 
atividade, porque articula uma necessidade a um objeto. Uma atividade envolve a realização de uma ou mais açóes, que por sua vez são compostas de operações.

Batista (2011) fez um levantamento bibliográfico, que revelou que a TA dá suporte a estudos sobre a formação do pensamento teórico e, em especial, pode ser utilizada como aporte teórico para a m-learning.

\section{OBJETIVO}

O objetivo deste artigo é apresentar uma revisão sistemática da literatura dos últimos 15 anos (2005 a 2019), que apresente estudos e resultados que abordem a aplicação da Aprendizagem Baseada em Problemas (ABP), da Modelagem Matemática (MM), da m-learning, da Teoria da Atividade (TA) e do uso de dispositivos móveis (smartphones) e softwares matemáticos no ensino e aprendizagem de CDI em cursos de Engenharia.

\section{REVISÃO SISTEMÁTICA DA LITERATURA}

A fim de apresentar o estado da arte, no que se refere a pesquisas em ABP, MM, TA, m-learning/b-learning e uso de softwares matemáticos no ensino e na aprendizagem de Cálculo Diferencial e Integral (CDI) nos cursos de Engenharia, utilizou-se a Revisáo Sistemática da Literatura (RSL). Conforme Kitchenham (2004), esta técnica que permite identificar, analisar e interpretar as evidências de um tema de pesquisa, cujas etapas são ilustradas na Figura 4.1. 
Figura 4.1 - Etapas da Revisão Sistemática da Literatura

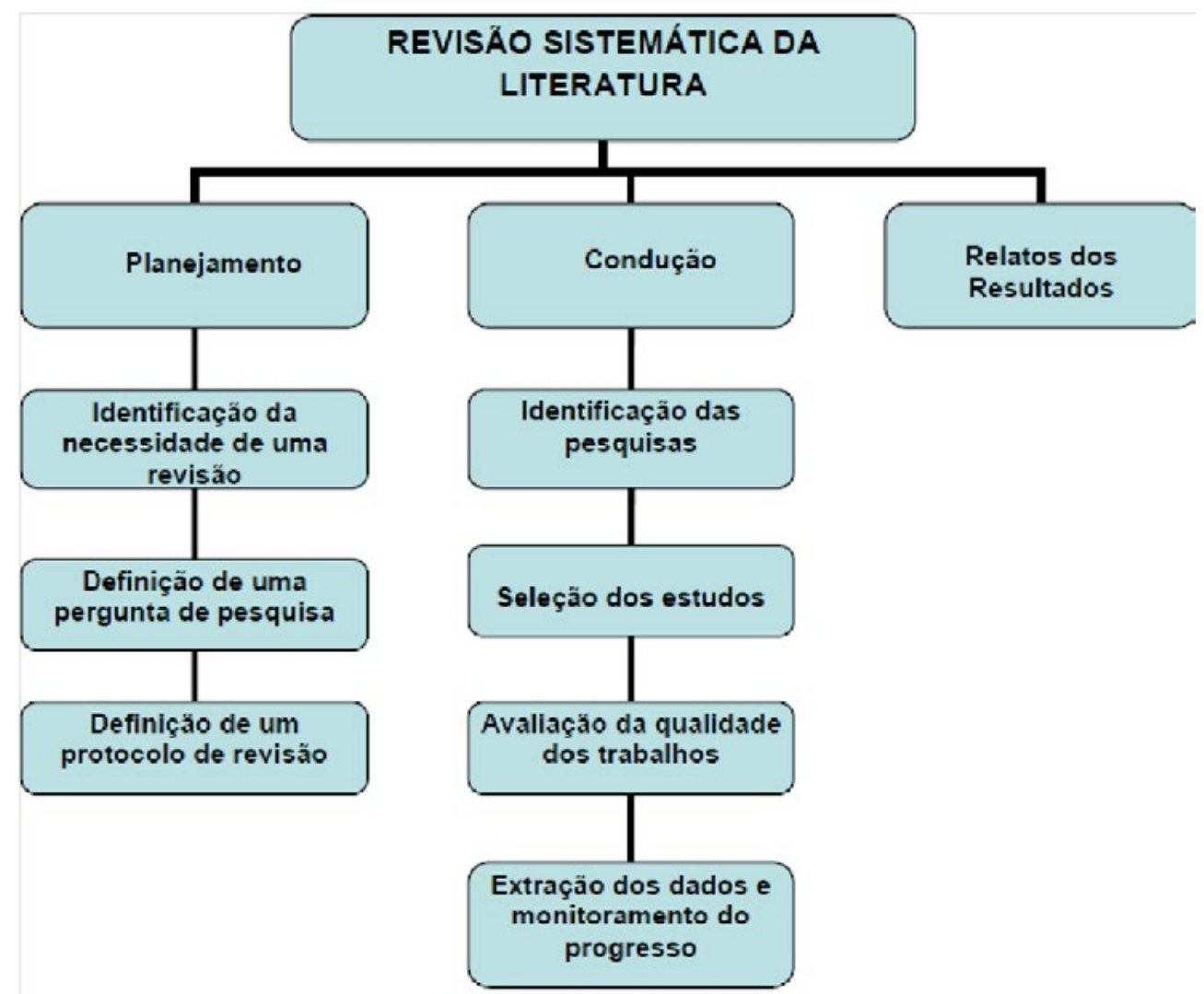

Fonte: Adaptado de Almeida (2016).

Kitchenham (2004) orienta que, embora as etapas indicadas anteriormente possam parecer sequenciais, é importante reconhecer que muitas delas envolvem interaçáo, como, por exemplo, a "definição do protocolo da revisão" e a "condução da revisão".

Para Kitchenham (2004), algumas razóes para se fazer uma RSL são:

- Identificar lacunas na pesquisa corrente, com a finalidade de sugerir áreas para investigaçóes mais profundas;

- Prover uma base ou arcabouço, com a intenção de adequadamente posicionar novas atividades de pesquisa;

- Examinar a influência de evidências empíricas, para corroborar ou contradizer hipóteses;

- Ou ainda auxiliar na geração de novas hipóteses. 


\subsection{Planejamento}

Devido ao fato de o m-learning, o uso da Teoria da Atividade (TA), da Modelagem Matemática (MM), da Aprendizagem Baseada em Problemas (ABP), de smartphones e de softwares matemáticos, no ensino e aprendizagem de Cálculo Diferencial e Integral (CDI), serem campos de pesquisa relativamente recentes, definiram-se as seguintes questóes:

- De que forma os dispositivos móveis, o m-learning e os apps matemáticos têm sido utilizados nas séries iniciais dos cursos de engenharia, no ensino e aprendizagem de CDI?

- De que forma a Aprendizagem Baseada em Problemas (ABP), Modelagem Matemática (MM) e a Teoria da Atividade (TA) tem sido usadas como aporte teórico no ensino e aprendizagem de Cálculo Diferencial e Integral?

De modo a responder essas questóes, a RSL deve também incluir estudos que abordem a utilização de m-learning, dispositivos móveis e apps matemáticos no Ensino Superior, com aporte teórico da ABP, da MM e da TA, nos componentes curriculares do cursos de Engenharia, que trabalham o ensino e a aprendizagem de Cálculo Diferencial e Integral, bem como dos impactos apresentados nestas experiências.

Definiu-se também um "protocolo de revisão", última etapa do planejamento, a qual define como se conduz a revisão sistemática da literatura. Este protocolo consistiu nas etapas descritas na sequência.

\subsubsection{Seleçáo das Bases de Consulta}

Loureiro (2012) indica que a busca seja feita em diversas fontes, a fim de reduzir a possibilidade de viés. Para este trabalho, as pesquisas foram realizadas nas bases de dados, ilustradas no Quadro 4.1.

Quadro 4.1 - Bases Pesquisadas

\begin{tabular}{|l|l|}
\hline \multicolumn{1}{|c|}{ Base } & \multicolumn{1}{c|}{ Descriçáa } \\
\hline $\begin{array}{l}\text { CAPES (Coordenaçáo de } \\
\text { Aperfeiçoamento de Pessoal de } \\
\text { Nível Superior) }\end{array}$ & $\begin{array}{l}\text { Agência de fomento à pesquisa brasileira que atua na } \\
\text { expansão e consolidaçáo da pós-graduação stricto sensu } \\
\text { (mestrado e doutorado) em todos os estados do país. A } \\
\text { CAPES divulga trabalhos científicos sob a forma de artigos, } \\
\text { teses e dissertaçóes. No caso específico dessa pesquisa, fizemos } \\
\text { a busca em Periódicos revisados por pares e no Banco de teses } \\
\text { e dissertaçóes da CAPES. }\end{array}$ \\
\hline $\begin{array}{l}\text { SCIELO (Scientific Eletronic } \\
\text { Library Online) }\end{array}$ & $\begin{array}{l}\text { Biblioteca eletrônica que proporciona um amplo acesso a } \\
\text { coleçós de periódicos científicos de vários países em texto } \\
\text { completo. }\end{array}$ \\
\hline Scholar Google (Google Acadêmico) & $\begin{array}{l}\text { Mecanismo de busca da Google, que concentra pesquisa em } \\
\text { trabalhos acadêmicos, tais como revistas científicas, livros, } \\
\text { teses, dissertaçóes, monografias, artigos e outros disponíveis } \\
\text { na Internet. }\end{array}$ \\
\hline
\end{tabular}




\begin{tabular}{|l|l|}
\hline \multicolumn{1}{|c|}{ Base } & \multicolumn{1}{c|}{ Descrição } \\
\hline $\begin{array}{l}\text { RBIE (Revista Brasileira de } \\
\text { Informática na Educação) }\end{array}$ & $\begin{array}{l}\text { Publica trabalhos desenvolvidos na área da Informática na } \\
\text { Educaçáo. }\end{array}$ \\
\hline $\begin{array}{l}\text { IEEE (Institute of Electrical and } \\
\text { Electronics Engineers) Xplore }\end{array}$ & $\begin{array}{l}\text { Biblioteca digital do IEEE, que fornece acesso a periódicos } \\
\text { do IEEE, revistas e atas de conferências. }\end{array}$ \\
\hline $\begin{array}{l}\text { RCAAP (Repositório Científico de } \\
\text { Acesso Aberto de Portugal) }\end{array}$ & $\begin{array}{l}\text { Portal financiado pelo Ministério da Ciência, Tecnologia e } \\
\text { Ensino Superior de Portugal. Visa promover o acesso aberto } \\
\text { à comunidade científica e acadêmica portuguesa, e aumentar } \\
\text { a visibilidade da investigação científica, sua acessibilidade e } \\
\text { divulgação. }\end{array}$ \\
\hline $\begin{array}{l}\text { ERIC Resources (Education } \\
\text { Information Center) }\end{array}$ & $\begin{array}{l}\text { Biblioteca on-line de educação e pesquisa em educação, } \\
\text { patrocinado pelo Institute of Education Sciences (IES) do } \\
\text { Departamento de Educação do governo dos Estados Unidos } \\
\text { da América. }\end{array}$ \\
\hline
\end{tabular}

Fonte: Próprios autores (2019).

\subsubsection{Definiçáo dos Termos de Busca}

Os termos de busca utilizados na busca foram "teoria da atividade", "activity theory", "modelagem matemática", "modelação matemática", "mathematical modeling", "mathematical modelling", "aprendizagem baseada em problemas", "problem-based learning", "pbl”, "abp", "m-learning", "mobile learning", "aplicativo", "app", "cálculo", "calculus", "engenharia”, "engineering" e combinaçôes diversas desses termos.

\subsubsection{Definiçáa de critérios para inclusão e exclusão dos estudos}

Para dar maior objetividade à busca e restringi-la, além dos termos de busca, foram definidos os critérios de inclusão e de exclusão de trabalhos de pesquisa, os quais são elencados a seguir.

Durante a busca, foram selecionados os trabalhos de pesquisa que atenderam os seguintes critérios de inclusáo:

a) Trabalhos que tenham sido publicados entre 2005 a 2019, tendo em vista se tratar de tema de pesquisa recente;

b) Trabalhos apresentados nos idiomas português ou inglês;

c) Trabalhos de pesquisa que incluíssem os termos de busca, indicados na subseção 4.1.2;

d) Trabalhos de pesquisa que relacionados ao ensino superior, particularmente no ensino e aprendizagem de CDI em cursos de Engenharia;

e) No caso de dispositivos móveis e m-learning, somente trabalhos de pesquisa relacionados com o uso de smartphones e apps matemáticos, no ensino e aprendizagem de CDI em cursos de Engenharia no Brasil e no Exterior; 
f) Trabalhos de pesquisa que se enquadrem no critério do item c) anterior e que apresentassem alguma avaliação experimental que apresentasse resultados parciais ou consolidados de pesquisa.

Não foram considerados, durante a busca, os trabalhos de pesquisa que se enquadrassem nos seguintes critérios de exclusáo:

a) $\mathrm{O}$ trabalho de pesquisa não está relacionado diretamente ao uso de smartphones e apps no ensino de CDI em cursos de Engenharia no Brasil ou no Exterior;

b) $\mathrm{O}$ trabalho de pesquisa apenas propóe/discute ou defende o uso de smartphones e apps no ensino e aprendizagem de CDI em cursos de engenharia no Brasil, mas não apresenta resultados (sejam parciais ou totais) de uma aplicação prática;

c) Trabalhos de pesquisa publicado em outros idiomas, além do português e inglês;

d) Trabalhos de pesquisa publicados fora do período investigado;

e) O trabalho de pesquisa não deixa claro o contexto em que é realizada a pesquisa, não informando, por exemplo, o nível de ensino investigado com o uso dos dispositivos móveis, e apenas apresenta de forma genérica o estudo realizado;

f) Trabalhos de pesquisa e artigos duplicados;

g) O público alvo do trabalho de pesquisa não foi do curso de engenharia, nem de disciplinas ou componentes curriculares diretamente relacionados com CDI.

\subsection{Conduçáo}

A busca, identificação e seleção dos estudos foram executadas via Internet, entre agosto de 2018 e maio de 2019, nas bases de dados citadas e eventualmente em outras fontes, usando as combinaçóes dos termos de busca, respeitados os critérios de inclusão e de exclusão indicados na seção anterior.

O principal mecanismo de busca via Internet, foi o "Google Acadêmico (Google Scholar)", uma vez que esse mecanismo também inclui, na busca, os demais repositórios mencionados na seção 4.1, desde que estivessem disponíveis na Internet.

Inicialmente, a busca foi concentrada em encontrar trabalhos de pesquisa, realizados com alunos de cursos de Engenharia presenciais, híbridos ou à distância, que:

a) Tivessem relação com o ensino e aprendizagem de disciplinas ou componentes curriculares, que diretamente ministrassem conteúdos de Cálculo Diferencial e Integral (CDI), levando em consideraçáo que estes conteúdos podem ser ministrados com diferentes formas de organização curricular, dependendo da Instituição de Ensino Superior; 
b) Envolvessem m-learning (baseado na TA) e o uso de dispositivos móveis (particularmente, smartphones);

c) Fossem baseados na combinação da Aprendizagem Baseada em Problemas (ABP) e Modelagem Matemática (MM);

d) Fossem publicados entre 2005 e 2019.

Cabe salientar que, conforme orientam Wohlin et al. (2012) e Barcelos (2014), em casos de mapeamento mais abrangentes da literatura, a definição rígida de critérios de qualidade não se torna essencial, porque se pode omitir a inclusão de estudos que possam apontar tendências deste campo de pesquisa. Assim, para esta RSL, não foram definidos critérios de exclusão com base na verificaçáo de qualidade.

A primeira busca, com a combinação de todos os termos, usando convenientemente os conectivos "E" (“AND") e "OU” (“OR"), no "Google Acadêmico" e nos demais repositórios, não encontrou teses, dissertaçóes ou artigos científicos, de acordo com os critérios de inclusão e de exclusão adotados, e que envolvessem simultaneamente Aprendizagem Baseada em Problemas, Modelagem Matemática, Teoria da Atividade, m-learning com o uso de dispositivos móveis (em particular, smartphones) e apps matemáticos. Tomou-se o cuidado de se utilizar os termos mais genéricos possíveis, de modo a ampliar o campo de busca.

O passo seguinte foi fazer a pesquisa com combinaçóes parciais dos termos de busca, com a finalidade de encontrar trabalhos de pesquisa similares, ou até na mesma linha. O resultado das buscas apontou um total de 82 trabalhos de pesquisa (comunicaçóes científicas, relatos de experiência, artigos, dissertaçóes e teses) que envolviam o ensino e aprendizagem de CDI em cursos de Engenharia, com aporte teórico da $\mathrm{ABP}$, da $\mathrm{TA}$ ou da $\mathrm{MM}$, com uso ou não de m-learning, dispositivos móveis e apps matemáticos, no período de 2005 a 2019. A Tabela 2.2 ilustra as quantidades de trabalhos encontrados durante a RSL, nas diferentes categorias.

\subsection{Resultados da RSL}

A RSL, desenvolvida neste trabalho de tese, além daqueles mostrados no Quadro 4.1, trouxe dados interessantes, que estão expostos nos parágrafos seguintes, sobre o uso de recursos de TIC, particularmente daqueles voltados ao ensino de CDI em cursos de Engenharia. 
Quadro 4.2 - Quantidades de estudos encontrados durante a RSL, de acordo com a metodologia de ensino de CDI em cursos de Engenharia.

\begin{tabular}{|c|c|c|}
\hline Uso de Softwares Matemáticos & Metodologias & Quantidade de Estudos \\
\hline \multirow{7}{*}{ SIM } & $\begin{array}{l}\text { Sem m-learning e sem aporte } \\
\text { teórico de ABP, MM ou TA. }\end{array}$ & 10 \\
\hline & $\begin{array}{l}\text { Com m-learning e sem aporte } \\
\text { teórico de ABP, MM ou TA. }\end{array}$ & 11 \\
\hline & Com ABP e m-learning. & 1 \\
\hline & Com TA e m-learning. & 3 \\
\hline & $\begin{array}{l}\text { Com MM, TA e Aprendizagem } \\
\text { Baseada em Projetos, com } \\
\text { m-learning (tablets e notebooks). }\end{array}$ & 1 \\
\hline & Com MM & 11 \\
\hline & Com TA & 2 \\
\hline \multicolumn{2}{|c|}{ Subtotal 1} & 39 \\
\hline \multirow{5}{*}{ NÂO } & $\mathrm{MM} \mathrm{e} \mathrm{ABP}$ & 1 \\
\hline & MM e TA & 3 \\
\hline & Somente ABP & 5 \\
\hline & Somente MM & 14 \\
\hline & Somente TA & 1 \\
\hline \multicolumn{2}{|c|}{ Subtotal 2} & 24 \\
\hline \multicolumn{2}{|c|}{ Revisões da Literatura } & 19 \\
\hline \multicolumn{2}{|c|}{ TOTAL } & 82 \\
\hline
\end{tabular}

Fonte: Próprios autores (2019).

Trinta e nove dos trabalhos levantados na pesquisa, que considerou trabalhos publicados entre 2005 e 2019, fazem mençáo ao uso de recursos de Tecnologias da Informação e Comunicação (TIC), a saber: softwares ou aplicativos matemáticos (35), planilhas eletrônicas de cálculo (3) e ambientes virtuais de aprendizagem (5), usados simultaneamente ou não. Três trabalhos não mencionaram especificamente um ou mais de um software ou aplicativo matemático, planilha eletrônica ou AVA. Desses 39 trabalhos de pesquisa, 15 foram dedicados ao estudo de $m$-learning e uso de dispositivos móveis. Por outro lado, 24 dos trabalhos de pesquisa encontrados na RSL dedicaram-se somente ao desenvolvimento e aplicaçáo de metodologias ativas, tais como a MM, a ABP e a TA, isoladamente ou combinadas entre si, sem a aplicaçáo de recursos das TIC. Finalmente, 19 deles se concentraram em revisóes da literatura dos temas citados.

Todos esses trabalhos de pesquisa, levantados durante a RSL, apresentaram grande preocupaçáo com os índices de retenção nos componentes curriculares que tratam de conteúdos de CDI e procuram alternativas para melhoria dos processos de ensino e de aprendizagem e, portanto de resultados melhores da aprendizagem 
dos alunos, o que implica em assimilar os conceitos de CDI de modo que tenham significado para os alunos e que possam utilizá-los para solucionar problemas do mundo real.

Observou-se, nos trabalhos pesquisados, pareceres favoráveis à aplicação dos recursos das TIC no ensino de conteúdos de CDI, principalmente com relação aos aspectos motivacionais, tendo em vista o uso de dispositivos móveis e modernos aplicativos que processam e resolvem expressóes algébricas que envolvam equaçôes, inequaçóes, limites, derivadas, integrais e equaçóes diferenciais, além de mostrar, de maneira prática e relativamente fácil, gráficos das funçóes matemáticas. As ressalvas e preocupaçôes foram relativas a determinadas restriçóes, tais como as capacidades de processamento e memória dos dispositivos móveis e à disponibilidade de acesso à Internet nos locais, onde os estudantes porventura conduzam seus estudos.

Conforme se pode ver no gráfico da Figura 4.2, no período pesquisado, a quantidade de trabalhos de pesquisa dedicados à aplicação das TIC, particularmente de m-learning e de aplicativos matemáticos, no ensino de CDI, em cursos de Engenharia, ainda é muito pequena, porém pode-se perceber uma tendência de crescimento, que pode até aumentar com uma maior popularização dos dispositivos móveis (em especial, os smartphones) e com o aprimoramento de suas características técnicas (velocidade de processamento e capacidade de armazenamento).

Figura 4.2 - Pesquisas realizadas sobre uso de recursos das TIC, de m-learning e de aplicativos matemáticos no ensino de CDI em cursos de Engenharia.

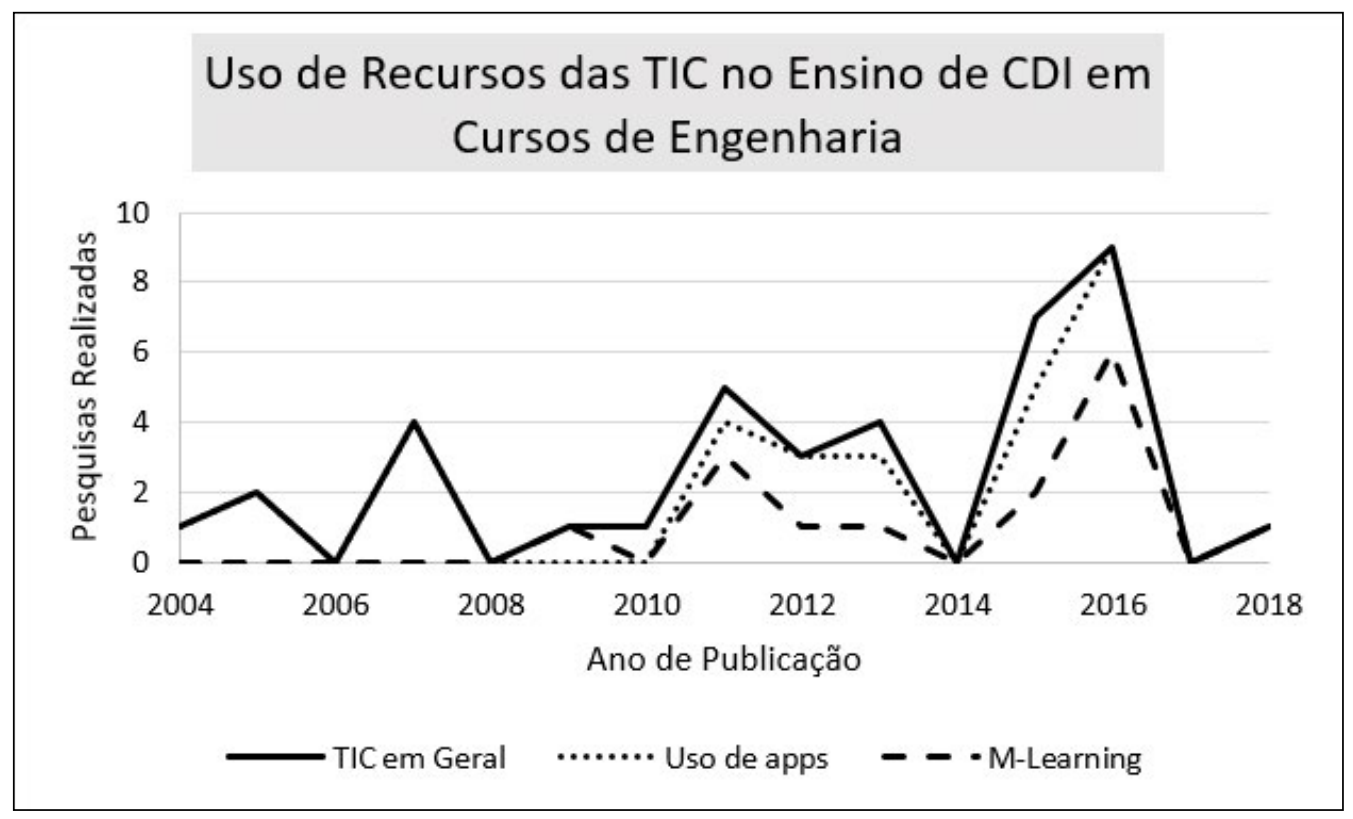

Fonte: Próprios autores (2019). 
Com relaçáo à escolha e ao uso de aplicativos nas pesquisas de ensino de CDI na Educação em Engenharia, no período pesquisado, percebeu-se uma crescente tendência de uso de softwares que tenham também versóes que possam ser instaladas e executadas em Sistemas Operacionais típicos de dispositivos móveis, tais como o "Android" e o "IOS". Na Figura 4.3, ilustram-se os aplicativos que mais apareceram nos trabalhos de pesquisa levantados durante a RSL.

Figura 4.3 - Ocorrências do uso de apps matemáticos, nos trabalhos de pesquisa de ensino de CDI nos cursos de Engenharia.

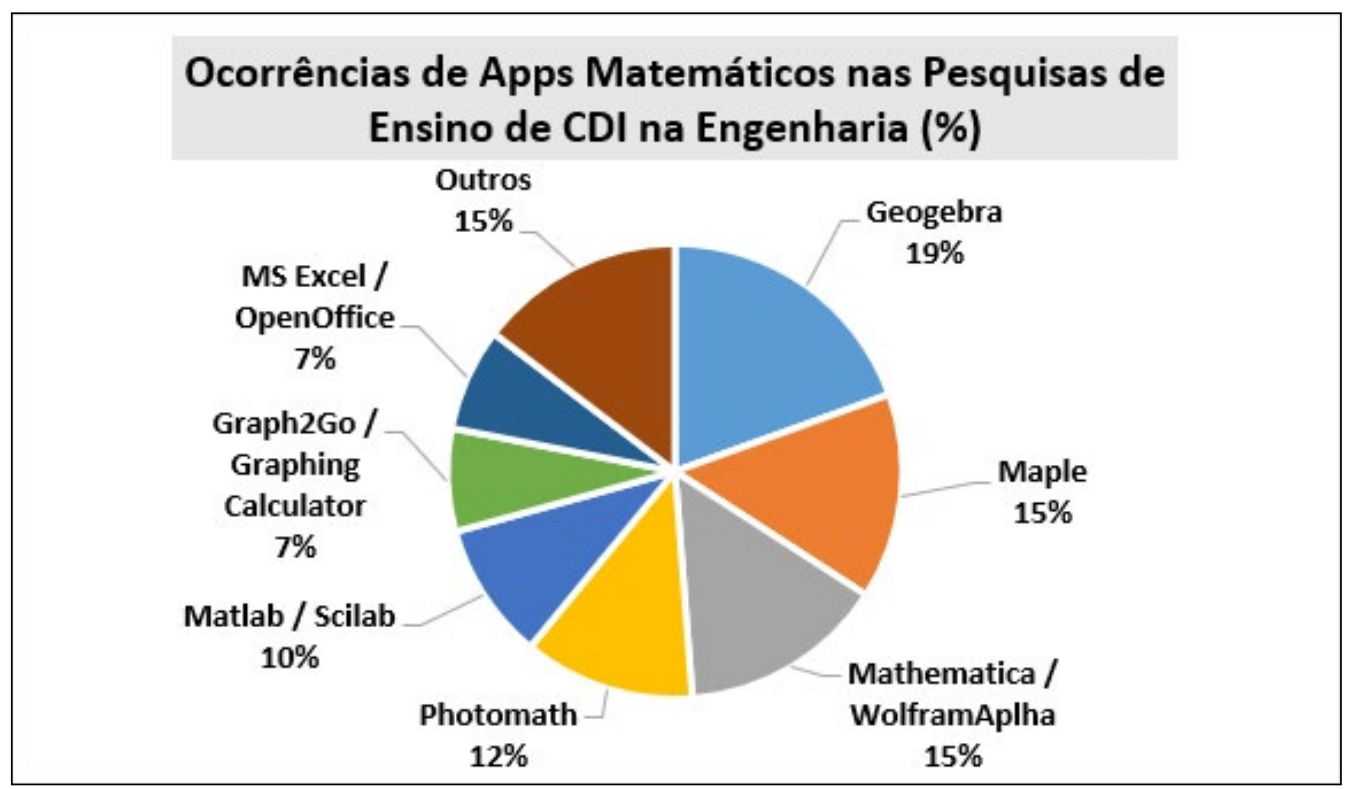

Fonte: Próprio autor.

Percebe-se, na análise do gráfico setorial da Figura 4.3, um relativo predomínio do uso do "Geogebra", do "Photomath", "Maple" e "Wolfram|Alpha", sendo os dois primeiros gratuitos, o que é uma característica a se considerar, pois não implica em custos adicionais de aquisição.

Com relação aos objetivos das pesquisas, que envolveram o uso de m-learning, dispositivos móveis e apps matemáticos no ensino de CDI na Engenharia, o Quadro 4.3 mostra os objetivos mais frequentes nesses trabalhos de pesquisa.

Quadro 4.3 - Objetivos mais pesquisados no ensino de CDI em cursos de Engenharia, que consideram a aplicação de m-learning, dispositivos móveis e apps matemáticos.

\begin{tabular}{|l|c|}
\hline \multicolumn{1}{|c|}{ Objetivos Pesquisados no Ensino de Conteúdos de CDI na Engenharia } & Ocorrências \\
\hline Analisar o uso e a influência do software. & 21 \\
\hline Analisar o uso de dispositivos móveis. & 9 \\
\hline
\end{tabular}




\begin{tabular}{|l|c|}
\hline \multicolumn{1}{|c|}{ Objetivos Pesquisados no Ensino de Conteúdos de CDI na Engenharia } & Ocorrências \\
\hline Investigar o uso de MM com recursos das TIC. & 3 \\
\hline Analisar o comportamento dos alunos e materiais usados em seus estudos. & 2 \\
\hline Testar metodologias de ensino. & 2 \\
\hline $\begin{array}{l}\text { Identificar processo de aprendizagem de conteúdos e averiguar os materiais que } \\
\text { foram ou são usados na aprendizagem. }\end{array}$ & 1 \\
\hline
\end{tabular}

Fonte: Próprios autores (2019).

Pode-se perceber, portanto, observando os dados do Quadro 4.3, uma quantidade significativa de pesquisas, que estudaram a aplicação de recursos das TIC no ensino superior, com destaque para o uso de dispositivos móveis.

Os trabalhos pesquisados, na RSL, possuem, como público alvo, alunos de cursos de Engenharia, principalmente aqueles que apresentam algum tipo de problema de aprendizagem em CDI, componente curricular que é um dos pilares da formação básica do engenheiro. Apesar de muitos trabalhos terem usado como aporte teórico a ABP, a MM, a TA, bem como estudarem a aplicação de recursos da m-learning e softwares matemáticos no ensino e aprendizagem de CDI em cursos de Engenharia, poucos combinaram essas teorias entre si e / ou com esses recursos tecnológicos. Somente trabalhos relativamente mais recentes começam a estudar o uso de smartphones (m-learning) em processos de ensino e aprendizagem, seja em cursos presenciais, semipresenciais ou à distância, o que se depreende da Figura 4.2.

Os dados supra permitem perceber a necessidade de se fazer mais pesquisas, envolvendo os temas tratados, principalmente no ensino superior e, especificamente, no ensino e aprendizagem de CDI em cursos de Engenharia. Há, portanto, ainda muito campo, para explorar a aplicação da ABP, da MM e da TA, seja isoladamente ou mesmo combinadas entre si, bem como incluindo ou não o uso de m-learning, dispositivos móveis e softwares matemáticos para o ensino e aprendizagem de CDI nos cursos de Engenharia.

\section{CONSIDERAÇÓESS FINAIS}

Há uma tendência crescente, nas pesquisas, em estudar a aplicação de recursos das TIC. Por outro lado, poucos trabalhos se utilizaram do aporte teórico da TA, da ABP e da MM e nenhum dos trabalhos levantados na RSL combina essas três teorias, com uso de recursos das TIC (com a aplicaçáo de m-learning, dispositivos móveis e apps matemáticos) no ensino de CDI em cursos de Engenharia.

Os autores deste artigo ressaltam o potencial que os recursos das TIC aqui tratados possuem, a fim de melhorar significativamente e tornar mais dinâmico o ensino e a aprendizagem dos conteúdos de CDI, tendo em vista a gama de bons resultados dos trabalhos já publicados sobre o tema. Porém, a aplicação desses recursos necessitam de um aporte teórico que a torne mais robusta, melhorando a motivação do estudante, oferecendo também a oportunidade para o aluno 
desenvolver suas habilidades de solucionar problemas (preferencialmente, situaçóesproblemas reais), assimilar e fixar os conceitos, de modo que possa reconhecer em que situaçóes ele aplicará um ou outro conceito, o que o possibilita a desenvolver consciência das vantagens, das desvantagens, da amplitude e dos limites inerentes ao uso dessa tecnologia.

A RSL desenvolvida trouxe a prospecçáo de pesquisas feitas nos últimos 15 anos, que envolvessem o estudo e a aplicação da Aprendizagem Baseada em Problemas, da Modelagem Matemática, da Teoria da Atividade, da m-learning e do uso de smartphones e softwares matemáticos, no ensino e aprendizagem de CDI no Ensino Superior, particularmente nos cursos de Engenharia, isoladamente ou combinadas entre si.

Conforme exposto, levantou-se um total de 82 pesquisas, das quais 63 trataram de aplicaçôes que envolveram alguma avaliação experimental, que apresentaram resultados parciais ou consolidados de pesquisa, tendo em vista se ter uma visão do estado da arte da pesquisa que envolvessem as metodologias e teorias supra citadas, tomando por referência as razóes a) e b) citadas por Kitchenham (2004).

Mostrou-se que a quantidade de pesquisas que investigam o uso das abordagens metodológicas ativas de ensino e de aprendizagem aqui tratadas, no ensino de Cálculo Diferencial e Integral em cursos de Engenharia, combinadas ou não com o uso de recursos tecnológicos, ainda é relativamente pequena. Por outro lado, mostrou-se também que há uma tendência de crescimento, na medida em que as tecnologias se tornem mais conhecidas e usuais, que se reconheça seu potencial de incrementar significativamente os processos de ensino e aprendizagem, sua popularizaçáo, a habilidade que os jovens possuem para lidar com as mesmas e um maior desenvolvimento de aplicativos específicos (BATISTA, 2011).

Quanto às dificuldades enfrentadas pelos alunos das séries iniciais dos cursos de Engenharia, os trabalhos desenvolvidos por Ferruzi (2003), Costa et al. (2005), Fecchio (2010), Bassanezi (2011), Pontes et al. (2012), Costa e Silva (2013), Ferruzzi e Almeida (2013), Carius e Ferreira (2014), Ortega, Lozano e Tristancho (2015), Silva, Jardim e Carius (2016), Souza (2016) e Guimarães (2018) apontaram para os altos índices de reprovação e retenção nas disciplinas de Cálculo, em geral logo no início dos cursos de Engenharia, e, em alguns casos, em disciplinas que necessitam dos conhecimentos naquelas desenvolvido (ZONTA e BONA, 2016; PONTES et al., 2012), causados por ausência de conhecimento e fracasso na ação pedagógica na construção de aprendizagens (ORTEGA, LOZANO e TRISTANCHO, 2015; FERRUZZI, 2003).

Os trabalhos sinalizam dificuldades dos alunos (COSTA et al., 2005; BATISTA, 2011; SILVA, 2013; SILVA, JARDIM e CARIUS, 2016), em compreender os conceitos matemáticos, devido à abstraçáo dos conteúdos, pouca destreza algébrica e falta de hábitos de estudo (MAGALHÁES, 2016; OKTAVYIANTHI e SUPRIANI, 2015), bem como com conceitos básicos e suas aplicações (JARDIM et al., 2015; OLIVEIRA e FERNANDES, 2016). Para Dullius, Veit e Araújo (2007), alunos chegam à universidade sem as habilidades e requeridas, para trabalhar com 
conteúdos matemáticos básicos, além de não demostrar interesse pelo conteúdo e não perceber a necessidade de adquirir esse conhecimento (BIEMBENGUT, HEIN e LOSS, 2010), para utilizá-lo na vida profissional.

Na mesma linha, Mokhtar et al. (2010) afirmam que, no modo tradicional, os conteúdos de CDI parecem não ter significado "real", sendo difíceis e abstratos; os professores usam um ensino mecanizado, com tipos padróes de problemas e soluções, com os alunos aprendendo de forma mecânica por repetição, o que, segundo Ferreira e Zuin (2018), os leva a um baixo desempenho. Caldeira (2014) aponta que os alunos questionam acerca da relevância de Cálculo e se mostram desmotivados, devido ao fato dos conteúdos serem de difícil compreensão e por não terem formação suficiente para aprendê-los. Finalmente, a falta de conexão da teoria com a prática faz com que os alunos considerem a Matemática irrelevante (HERNANDEZ-MARTINEZ e VOS, 2018) e a dissociação das disciplinas básicas dos cursos de Engenharia com relação às profissionalizantes causa impacto negativo na motivação (DANDOLINI, VANINI e SOUZA, 2004).

\section{REFERÊNCIAS}

ALMEIDA, Rosiney Rocha. Mobile Learning no Processo de Ensino e Aprendizagem de Conteúdos de Genética: proposta e análise com base na Teoria da Atividade. Tese (Doutorado em Ensino de Ciências e Matemática) - UNICSUL - Universidade Cruzeiro do Sul, São Paulo, 2016.

BARCELOS, T. S. Relaçóes Entre o Pensamento Computacional e a Matemática em Atividades Didáticas de Construçáo de Jogos Digitais. 2014. Tese (Doutorado em Ensino de Ciências e Matemática) - Universidade Cruzeiro do Sul, São Paulo, 2014.

BASSANEZI, Rodney Carlos. Ensino-Aprendizagem com Modelagem Matemática: uma nova estratégia. 3. ed. São Paulo: Contexto, 2011.

BATISTA, Silvia Cristina Freitas. MLearnMat: Modelo Pedagógico para Atividades de M-Learning em Matemática. 2011. 225 f. Tese (Doutorado em Informática na Educação) - Universidade Federal do Rio Grande do Sul, Porto Alegre, 2011. Disponível em: <https://www.lume.ufrgs.br/handle/10183/48916>. Acesso em: 20 Mar. 2019.

BIEMBENGUT, Maria Salett; HEIN, Nelson. Modelagem Matemática no Ensino. 5 Ed. São Paulo: Contexto, 2009. Disponível em: <https://bv4.digitalpages. com.br/?term=Modelagem\%2520Matem\%25C3\%25A1tica\%2520no\%2520Ensino\&searchpage $=1 \&$ filtro $=$ todos $\&$ from $=$ busca\&page $=4 \&$ section $=0 \# /$ legacy $/ 1546>(\mathrm{Bi}-$ blioteca Virtual da Universidade Cruzeiro do Sul). Acesso em: 11 jun. 2019.

BIEMBENGUT, Maria Salett; HEIN, Nelson; LOSS, Gabriel Schneider. Modelagem Matemática no Ensino de Matemática na Engenharia. 2010. Disponível em: <http:// www.pucrs.br/edipucrs/erematsul/comunicacoes/21GABRIELSCHNEIDERLOSS.pdf>. Acesso em 20 mar. 2019. 
CALDEIRA, Rutyele Ribeiro. Cálculo em Ação, Modelagem e Parcerias: possibilidades para aprendizagens expansivas em um contexto de formação em Engenharias. 2014. 229 f. Tese (Doutorado em Educação) - Universidade Federal de Minas Gerais, Belo Horizonte, 2014. Disponível em: <http://www.bibliotecadigital.ufmg.br/dspace/handle/1843/BUOS9RPN3K>. Acesso em: 25 abr. 2019.

CARIUS, Ana Carolina; FERREIRA, Gessé Pereira. O Cálculo Diferencial e Integral e Suas Dificuldades: uma abordagem interdisciplinar para estudantes de Engenharia. In: Congresso Iberoamericano de Ciencia, Tecnologia, Inovación y Educación, Buenos Aires, 2014. Disponível em: <https://www.oei.es/historico/congreso2014/ memoriactei/290.pdf>. Acesso em: 29 abr. 2019.

COSTA, Luciano Andreatta Carvalho da; BRUSCHI, Diogo Lino; MUGGE, Tobias Roberto; MEIROSE, Lucas. O Estudo das Equaçóes Diferenciais Utilizando a Modelagem Matemática em um Curso de Engenharia. Anais do XXXIII Congresso Brasileiro de Educaçáo em Engenharia - COBENGE, Campina Grande, 2005. Disponível em: <http://www.abenge.org.br/cobenge/arquivos/14/artigos/RS-560748079068-1119043455999.pdf>. Acesso em: 14 mar. 2019.

COSTA, Samira Domingos; SILVA, Viviane Cota. A Derivada como Taxa de Variação em um Curso de Engenharia Elétrica: imagem conceitual e definição conceitual. Proceedings of VII International Conference on Engineering Education, Luanda, 2013. Disponível em: <http://copec.eu/congresses/icece2013/proc/works/70.pdf>. Acesso em: 24 jan. 2019.

DANDOLINI, G. A.; VANINI, L.; SOUZA, J. A. A Utilização de Software no Ensino de Cálculo. In: XXXII Congresso Brasileiro de Educação em Engenharia, Brasília. Anais do XXXII COBENGE, 2004. Disponível em: <http://www.abenge.org.br/cobenge/ arquivos/15/artigos/01_463.pdf>. Acesso em: 11 abr. 2019.

DULLIUS, Maria Madalena; VEIT, Eliane A.; ARAÚJO, Ives S. Uso de Recursos Computacionais para O Ensino e Aprendizagem de Equações Diferenciais. In: Anais do XXXV Congresso Brasileiro de Educação em Engenharia - COBENGE 2007, Curitiba, p. 3B07-1, 3B07-15, 2007. Disponível em: <http://www.abenge.org.br/ cobenge/arquivos/12/artigos/268-Maria\%20Madalena\%20Dullius.pdf $>$. Acesso em: 27 abr. 2019.

FECCHIO, Roberto. A Modelagem Matemática Como Recurso Didático em Projetos Interdisciplinares. Unión - Revista Iberoamericana de Educación Matemática, n. 22, p. 133-145, junho - 2010. Disponível em: <http:/www.fisem.org/www/union/ revistas/2010/22/Union_022_013.pdf>. Acesso em: 14 mai. 2019.

FERREIRA, Allan Silva; ZUIN, Elenice de Souza Lodron. Introdução do Conceito de Derivada a Partir da Investigação Matemática. Revista BoEM, Joinville, v. 6, n. 10, p. 82-102, 2018. Disponível em: <http://200.19.105.203/index.php/boem/article/ view/11731>. Acesso em: 18 Abr. 2019. 
FERRUZZI, Elaine Cristina. A Modelagem Matemática como Estratégia de Ensino e Aprendizagem do Cálculo Diferencial e Integral nos Cursos Superiores de Tecnologia. 2003. 154 f. Dissertação (Mestrado em Engenharia de Produção) - Universidade Federal de Santa Catarina, São Paulo, 2003. Disponível em: <http://repositorio.ufsc.br/xmlui/ handle/123456789/84624>. Acesso em: 12 mar. 2019.

FERRUZZI, Elaine Cristina; ALMEIDA, Lourdes Maria Werle de. Modelagem Matemática no Ensino de Matemática para Engenharia. RBECT, v. 6, n. 1, 2013. Disponível em: <https:/www.researchgate.net/profile/Lourdes_Almeida2/ publication/273995161_Modelagem_Matematica_no_ensino_de_Matematica_para_ engenharia/links/55f6f18c08aeba1d9eeda556/Modelagem-Matematica-no-ensino-deMatematica-para-engenharia.pdf>. Acesso em: 10 jan. 2019.

GRYMUZA, Alissá Mariane Garcia. REGO; Rogéria Gaudêncio do. Teoria da Atividade: uma possibilidade no ensino de matemática. Revista Temas em Educação, João Pessoa, v.23, n.2, p. 117-138, jul.-dez. 2014. Disponível em: <https://periodicos.ufpb.br/index. php/rteo/article/view/20864/12564>. Aceso em: 14 jan. 2019.

GUIMARÃES, Gilselene. Aprendendo Cálculo Diferencial e Integral em Engenharia Civil: Uma Proposta Interdisciplinar Entre Teoria e Prática. Revista de Ensino de Engenharia. Brasília, v. 37, n. 1, p. 66-75, 2018. Disponível em: <http://revista.educacao. ws/revista/index.php/abenge/article/view/1288>. Acesso em: 15 fev. 2019. <DOI: 10.5935/2236-0158.20180007>.

HERNANDEZ-MARTINEZ, Paul; VOS, Pauline. "Why Do I Have to Learn This?” A Case Study on Students' Experiences of The Relevance of Mathematical Modelling Activities. ZDM Mathematics Education, v. 50, n. 1-2, p. 245-257, 2018. Disponível em: <https://doi.org/10.1007/s11858-017-0904-2>. Acesso em: 12 abr. 2019.

JARDIM, Deborah Faragó; SILVA, Jaqueline Maria da; PEREIRA, Marcela Marins; SOARES JR., Eduardo Antônio; NEPOMUCENA, Thâmara Vieira; PINHEIRO, Thaís Rodrigues. Estudando Limites com Geogebra. Revista Científica Vozes dos Vales, n. 8, ano 4, p. 1-19, 2015. Disponível em: <https://www.researchgate.net/profile/ Jaqueline_Da_Silva5/publication/286220543_Estudando_Limites_com_o_Geogebra/ links/5666e68908aef42b57867571/Estudando-Limites-com-o-Geogebra.pdf>. Acesso em: 28 mar. 2019.

JAWORSKI, Barbara. Mathematical Understanding and its Relation to Design of Teaching. Proceedings of CERME8 - Eighth Congress of The European Society for Research in Mathematics Education, Manavgat-Side, 2013. Disponível em: <http://cerme8.metu. edu.tr/wgpapers/WG14/WG14_Jaworski.pdf>. Acesso em: 04 mai. 2019.

JAWORSKI, Barbara; PORTARI, Despina; PETROPOULOU, Georgia. Theorising University Mathematics Teaching: the teaching triad within an activity theory perspective. Proceedings of CERME10 - Tenth Congress of The European Society for Research in Mathematics Education, Dublin, 2017. Disponível em: <https://hal.archives-ouvertes.fr/ hal-01941348/document>. Acesso em: 04 mai. 2019. 
KIDRON, Ivy. Calculus Teaching and Learning. In: Lerman S. Encyclopedia of Mathematics Education. Dordrecht: Springer, 2014. Disponível em: <https://link. springer.com/referenceworkentry/10.1007\%2F978-94-007-4978-8_18\#howtocite>. Acesso em: 18 dez. 2018.

KITCHENHAM, Barbara. Procedures for Performing Systematic Reviews. Keele, Keele University, UK, v. 33, n. 2004, p. 1-28, 2004. Disponível em: <http://www.inf.ufsc. br/ aldo.vw/kitchenham.pdf>. Acesso em: 21 jan. 2019.

LEONTIEV, A. N. Activity, Consciousness and Personality. Englewood Cliffs: PrenticeHall, 1978. Disponível em: <https://www.marxists.org/archive/leontev/works/1978/ activity-consciousness-personality.pdf>. Acesso em: 16 mar. 2019.

LEONTIEV, A. N. O Desenvolvimento do Psiquismo. 2 Ed. São Paulo: Centauro, 2004. Disponível em: <https://www.academia.edu/22546933/O_desenvolvimento_do_ psiquismo._Leontiev_Alexis>. Acesso em: 15 mar. 2019.

LOUREIRO, S. A. Revisão Sistemática da Literatura. Laboratório de Aprendizagem em Logística e Transportes - LALT. Campinas: UNICAMP, 2012.

MAGALHÃES, José Manuel da Mota. Aprendizagem de conteúdos matemáticos de cálculo por alunos do $1 .^{\circ}$ ano de cursos de engenharia: contextos e materiais didáticos. 2016. Tese (Doutorado em Ciências da Educação - Educação Matemática) - Universidade do Minho - Instituto de Educação, 2016. Disponível em: <https:// repositorium.sdum.uminho.pt/handle/1822/46021>. Acesso em: 26 abr. 2019.

MOKHTAR, Mohd Zin; TARMIZI, Rohani Ahmad; AYUB, Ahmad Fauzi Mohd; TARMIZI, Mohd Ariff Ahmad. Enhancing Calculus Learning Engineering Students Through Problem-Based Learning. WSEAS Transactions on Advances in Engineering Education, v. 7, n. 8, 2010. Disponível em: <https:/www.researchgate.net/ publication/268291357_Enhancing_Calculus_Learning_Engineering_Students_ Through_Problem-Based_Learning $>$. Acesso em: 27 mar. 2019.

OKTAVIYANTHI, Rina; SUPRIANI, Yani. Utilizing Microsoft Mathematics in Teaching and Learning Calculus. In: Indonesian Mathematical Society Journal on Mathematics Education, v. 6, n. 1, p. 63-76, 2015. Disponível em: <https://eric. ed.gov/?id=EJ1079603>. Acesso em: 29 abr. 2019.

OLIVEIRA, Michelli Silva de; FERNANDES, Kleber Tavares. Uso de Aplicativos Móveis no Ensino da disciplina de Cálculo Diferencial e Integral. In: Anais do CTRL+e 2016 - Congresso Regional Sobre Tecnologias na Educaçáo, Natal, p. 127-138, 2016. Disponível em: <http://ceur-ws.org/Vol-1667/CtrlE_2016_AC_paper_30.pdf>. Acesso em: 14 mar. 2019.

ORTEGA, Mawency Vergel; LOZANO, José Joaquín Martínez; TRISTANCHO, Sandra Liliana Zafra. Apps en el Rendimiento Académico y Autoconcepto de Estudiantes de Ingeniería. Revista, Logos Ciencia \& Tecnologia, Bogotá, v. 6, n. 2, p. 198-208, 2015. Disponível em: <https://www.redalyc.org/pdf/5177/517751486005.pdf>. Acesso em: 20 mai. 2019. 
PIRES, Luiz Fernando Rodrigues; ESCHER, Marco Antônio. As Influências das Tecnologias da Informação E Comunicaçáo nas Estratégias de Ensino E Aprendizagem de Professores E Estudantes de Cálculo Diferencial E Integral. Rio de Janeiro: UFRJ, 2015. Disponível em: <http://www.uff.br/ emem/files/2015/10/AS-INFLU\%C3\%8ANCIAS-DAS-TECNOLOGIAS-DAINFORMA $\%$ C3\%87\%C3\%83O-E-COMUNICA\%C3\%87\%C3\%83O-NASESTRAT\%C3\%89GIAS-DE-ENSINO-E-APRENDIZAGEM-DE-PROFESSORESE-ESTUDANTES-DE-C\%C3\%81LCULO-DIFERENCIAL-E-INTEGRAL.pdf>. Acesso em: 16 mar. 2019.

PONTES, Pericles Crisiron; RIBEIRO, Maria do Socorro Souza; PEREIRA, Maria Juliana; FONSECA, Maria da Conceição Pereira; FONSECA, Maria Líbia Pereira. A Relação do Conhecimento de Cálculo I no Desempenho e Conclusão dos Cursos de Engenharia - Um Estudo de Caso no Curso de Alimentos. In: Anais do XL Congresso Brasileiro de Educação em Engenharia - COBENGE, Belém, 2012. Disponível em: <http://www.abenge.org.br/cobenge/arquivos/7/artigos/104411.pdf>. Acesso em: 13 out. 2019.

PREVOT, Fulvio Bianco; SCHIMIGUEL, Juliano. Aplicativos Matemáticos: podem ou não ajudar o processo de aprendizagem? In: Anais do XII ENEM - Encontro Nacional de Educação Matemática, São Paulo, 2016. Disponível em: <http://www.sbembrasil.org. br/enem2016/anais/pdf/7181_2878_ID.pdf>. Acesso em: 20 ago. 2017.

PREVOT, Fulvio Bianco. Uma Abordagem com Uso de M-Learning na Aprendizagem de Cálculo Diferencial e Integral em Cursos de Engenharia

Baseada em ABP e Modelagem Matemática. 2019. 221 p. Tese (Doutorado em Ensino de Ciências e Matemática) - Universidade Cruzeiro do Sul, São Paulo, 2019. Disponível em: <repositório.cruzeirodosul.edu.br/handle/123456789/282>. Acesso em: 17 jun. 2020.

RENZ JR., Herton. A Importância da Modelagem Matemática no EnsinoAprendizagem. 2015. 62 f. Dissertação (Mestrado Profissional em Matemática) Universidade Federal de Goiás, Catalão, 2015. Disponível em: <https://repositorio.bc.ufg. br/tede/bitstream/tede/4706/5/Disserta\%C3\%A7\%C3\%A3o\%20-\%20Herton\%20 Renz\%20J\%C3\%BAnior\%20-\%202015.pdf>. Acesso em: 10 abr. 2019.

RIBEIRO, Luís Roberto de Camargo; ESCRIVÃO FILHO, Edmundo; MIZUKAMI, Maria da Graça N. Uma Experiência com A PBL no Ensino de Engenharia Sob A Ótica dos Alunos. Anais do XXXI COBENGE - Congresso Brasileiro de Educaçáo em Engenharia. Rio de Janeiro: COBENGE, 2003. Disponível em: <http://www.abenge.org. br/cobenge/arquivos/16/artigos/NMT221.pdf>. Acesso em: 12 mar. 2019.

RIBEIRO, Luís Roberto de Camargo. Aprendizagem baseada em problemas (PBL): uma implementação na educação em engenharia na voz dos atores. Tese (Doutorado em Educação) - Universidade Federal de São Carlos, São Carlos, 2005. 
Disponível em: <https://repositorio.ufscar.br/bitstream/handle/ufscar/2353/TeseLRCR. pdf?sequence=1\&isAll>. Acesso em: 19 mar. 2019.

RIBEIRO, Luís Roberto de Camargo. Aprendizagem Baseada em Problemas (PBL) na Educação em Engenharia. Revista de Ensino de Engenharia. Brasília, v. 27, n. 2, p. 23-32, 2008. Disponível em: <https:/www.researchgate.net/profile/Luis_Ribeiro21/ publication/268183847_APRENDIZAGEM_BASEADA_EM_PROBLEMAS_ PBL_NA_EDUCACAO_EM_ENGENHARIA/links/568f18cf08aef987e567ef12/ APRENDIZAGEM-BASEADA-EM-PROBLEMAS-PBL-NA-EDUCACAO-EMENGENHARIA.pdf>. Acesso em: 15 mar. 2019.

SILVA, Carlos Antônio da. Introdução ao Conceito de Integral de Funçóes Polinomiais em Um Curso de Engenharia de Produçáo Por Meio de Tarefas Fundamentadas em Princípios da Modelagem Matemática. 2013. 349 p. Tese (Doutorado em Educação Matemática) - PUC/SP - Pontifícia Universidade Católica de São Paulo, São Paulo, 2013. Disponível em: <https://tede.pucsp.br/handle/ handle/10960>. Acesso em: 05 mai. 2019.

SILVA, Jaqueline Maria da; JARDIM, Deborah Faragó; CARIUS, Ana Carolina. O Ensino e A Aprendizagem de Conceitos de Cálculo Usando Modelos Matemáticos. Revista de Ensino de Engenharia, v. 35, n. 2, p. 70-80, 2016. Disponível em: <https:// www.researchgate.net/profile/Jaqueline_Da_Silva5/publication/312136068_O_ Ensino_e_a_Aprendizagem_de_Conceitos_de_Calculo_Usando_Modelos_ Matematicos_e_Ferramentas_Tecnologicas/links/5874221a08ae329d621d399e/OEnsino-e-a-Aprendizagem-de-Conceitos-de-Calculo-Usando-Modelos-Matematicos-eFerramentas-Tecnologicas.pdf>. Acesso em: 16 mai. 2019.

SOUZA, Debora Vieira de. O Ensino de Noçóes de Cálculo Diferencial e Integral por Meio da Aprendizagem Baseada em Problemas. 2016. 159f. Dissertação (Mestrado em Ensino de Ciências e Matemática) - IFSP - Instituto Federal de Educação Ciências e Tecnologia de São Paulo. São Paulo, 2016. Disponível em: <https://spo. ifsp.edu.br/images/phocadownload/DOCUMENTOS_MENU_LATERAL_FIXO/ POS_GRADUA\%C3\%87\%C3\%83O/MESTRADO/Ensino_de_Ci\%C3\%AAncias_e_ Matem\%C3\%A1tica/Dissertacoes/O-Ensino-de-No\%C3\%A7\%C3\%B5es-deC\%C3\%A1lculo-Diferencial-e-Integral-por-meio-da-Aprendizagem-Baseada-emProblemas-Disserta\%C3\%A7\%C3\%A3o-e-Produto-Final.pdf>. Acesso em: 27 abr. 2019.

SOUZA, Roberto Eugenio; SCHIMIGUEL, Juliano. A Importância da Metodologia PBL - Aprendizagem Baseada em Problemas - no Aprendizado dos Alunos e na Formação dos Professores. Anais do Encontro de Produçáo Discente em Ensino de Ciências e Matemática. São Paulo: PUC-SP / Universidade Cruzeiro do Sul, p. 1-10, 2014. Disponível em: <http://revistapos.cruzeirodosul.edu.br/index.php/epd/article/ view/976/757>. Acesso em: 23 fev. 2019.

VIGOTSKII, Lev Semenovich; LURIA, Alexander Romanovich; LEONTIEV, Alexis N. Linguagem, Desenvolvimento e Aprendizagem. Tradução de Maria da Pena Villalobos. Coleção Educação Crítica. 11 Ed. São Paulo: Ícone, 2010. Disponível em: <https://www. 
unifal-mg.edu.br/humanizacao/wp-content/uploads/sites/14/2017/04/VIGOTSKI-LevSemenovitch-Linguagem-Desenvolvimento-e-Aprendizagem.pdf>. Acesso em: 15 mar. 2019.

WOHLIN, C. et al. Experimentation in software engineering. Springer Science \& Business Media, 2012.

ZONTA, Giovani Renato; BONA, Juliano. Modelagem Matemática como Proposta para O Ensino de Cálculo nos Cursos de Engenharia. Revista Maiêutica, Indaial, v. 2, n. 1, p. 37-51, 2016. Disponível em: <https://publicacao.uniasselvi.com.br/index.php/ENG_ EaD/article/view/1525>. Acesso em: 15 mai. 2019. 